\title{
Quelques paramètres physiologiques et constituants biochimiques des organes de la lentille de terre (Macrotyloma geocarpum) en conditions de stress hydrique
}

\author{
Mberdoum Memti NGUINAMBAYE ${ }^{1 *}$, Rasmata NANA ${ }^{2}$, I. Alain DJINET ${ }^{3}$ et \\ Zoumbiessé TAMINI ${ }^{2}$
}

\author{
${ }^{1}$ Université de Ndjamena, Laboratoire de Botanique systématique et d'Ecologie Végétale BP 1027 \\ ${ }^{2}$ Laboratoire de Biosciences, Université Joseph KI-ZEEBO, 03 BP 7023 Ouagadougou 03, Burkina Faso. \\ ${ }^{3}$ Ecole Normale de Bongor. \\ *Auteur correspondant, E-mail: memti2020@gmail.com
}

\section{RESUME}

La lentille de terre est une espèce en voie de disparition car sa culture demeure rudimentaire et traditionnelle. Aucun effort n'est fait pour améliorer cette culture. C'est pourquoi, elle n'occupe pas une place particulière en Afrique comme d'autres cultures vivrières. Ainsi, l'étude a consisté d'une part, à évaluer l'effet de stress sur le taux d'accumulation des composés biochimiques, et d'autre part, à évaluer les paramètres physiologiques et agronomiques dans les organes. Pour cela, 3 niveaux d'alimentation hydrique sont considérés : un stress hydrique sévère de 12 jours, un autre léger de 7 jours et une alimentation normale de 2 jours. L'expérimentation a été conduite en serre dans des pots avec un sol sablonneux. Un seul essai a été mis en place pour une durée de 125 jours. Des résultats obtenus, il ressort que le stress appliqué a perturbé sérieusement les plantes sous stress sévère. La chlorophylle est plus concentrée dans les feuilles des plantes stressées les rendant plus foncées. Un décalage de début de floraison et de cycle est observé chez les plantes stressées. Les plantes sévèrement stressées présentent des gousses de taille 2 fois plus grosses $(3,5 \mathrm{~cm})$ que celles des plantes témoins $(1,8 \mathrm{~cm})$. Les plantes stressantes ont des organes produisant une biomasse sèche plus faible que ceux des plantes témoins. Les taux des glucides et des protéines sont élevés dans les racines et les feuilles. L'étude a montré que la lentille de terre est riche en glucide et en protéine. Le stress a d'effet sur le rendement et la qualité des gousses. Il est judicieux d'envisager la mesure du degré de tolérance à la sécheresse.

(C) 2020 International Formulae Group. All rights reserved.

Mots clés : Déficit hydrique, organes, Macrotyloma geocarpum, taux d'accumulation, biomasse.

\section{Some physiological and constituent parameters biochemical of the bodies of the ground lens (Macrotyloma geocarpum) in conditions of hydrous stress}

\begin{abstract}
The duckweed is an endangered species because its culture remains rudimentary and traditional. No effort is made to improve this culture. This is why it does not occupy a special place in Africa like other food crops. Thus, the study consisted, on the one hand, of evaluating the effect of stress on the rate of accumulation of biochemical compounds, and on the other hand, of evaluating the physiological and agronomic parameters in the
\end{abstract}


organs. For this, 3 levels of water supply are considered: a severe water stress of 12 days, another mild of 7 days and a normal water supply of 2 days. The experiment was carried out in a greenhouse in pots with sandy soil. A single trial was set up for a period of 125 days. From the results obtained, it appears that the stress applied has seriously disturbed plants under severe stress. Chlorophyll is more concentrated in the leaves of stressed plants, making them darker. A delayed start of flowering and cycle is observed in stressed plants. The severely stressed plants have pods twice as large $(3.5 \mathrm{~cm})$ as those of the control plants $(1.8 \mathrm{~cm})$. Stressful plants have organs producing a lower dry biomass than those of control plants. Carbohydrate and protein levels are high in the roots and leaves. The study showed that the ground lentil is rich in carbohydrates and proteins. Stress affects the yield and quality of the pods. It is a good idea to consider measuring the degree of drought tolerance.

(C) 2020 International Formulae Group. All rights reserved.

Keywords: Hydrous deficit, bodies, Macrotyloma geocarpum, rate of accumulation biomass.

\section{INTRODUCTION}

En Afrique, les céréales et les légumineuses constituent une source d'alimentation importante (Falalou, 2000). L'alimentation des peuples subsahariens est essentiellement basée sur les céréales et les légumineuses. Parmi ces légumineuses, la lentille de terre [(Macrotyloma geocarpum (Harms)], légumineuse géocarpe n'a pas une valeur alimentaire moindre. La lentille de terre est une légumineuse à graines mineures mais d'une importance alimentaire et nutritionnelle énorme. C'est l'une des légumineuses la moins connue, mais contribuant de façon significative à la nutrition en milieu rural, aux moyens de subsistance et au développement durable (Bampuori, 2007; Arernu et al., 2006). C'est une légumineuse annuelle cultivée surtout pour ses graines comestibles (Borus et Bosch, 2007 ; Adu- Gyamfi et al., 2011 ; Bayorbor et al., 2010). Sa consommation prend de plus en plus d'ampleur mais sa productivité demeure médiocre (moins de $500 \mathrm{~kg} / \mathrm{ha}$ ) (Kouelo, 2015). Elles offrent d'énormes potentialités notamment sur le plan nutritionnel par la fourniture de protéines alimentaires végétales accessibles à moindre coût et dans la gestion de la fertilité des sols (Kouelo et al.,2015). La culture constitue une importante source de glucides, de protéines et de minéraux et est donc utilisée pour compléter l'alimentation des ménages (Achigan et Vodouhè, 2006). En plus de son importance nutritionnelle, la lentille de terre est également adaptée aux conditions de sols pauvres (Adéranti, 2001). Les graines de la lentille de terre ont aussi des valeurs traditionnelles considérables.
La lentille de terre, espèce cultivée en Afrique de l'Ouest, est une espèce végétale en voie de disparition de nos jours. Les causes de cette disparition progressive concomitante d'une érosion génétique sont multiples (Amujoyegbe et al, 2012). Malheureusement, aucun effort n'est fait pour améliorer la culture de la lentille de terre. La culture demeure rudimentaire et traditionnelle. Malgré l'importance de la lentille de terre, peu de données sont disponibles sur la propagation de la culture, sa biodiversité, ses caractéristiques agronomiques, son potentiel de rendement, ses activités de fixation de l'azote, ses maladies et les ravageurs (Amujoyegbe et al, 2012). En effet, la lentille de terre fait partie des cultures sous-utilisées et négligées car aucun effort n'est fait localement ou internationalement pour apporter cette culture dans le développement scientifique, mais plutôt, elle est limitée aux agriculteurs locaux. Néanmoins, Ces cultures mineures jouent cependant un rôle crucial dans la sécurité alimentaire, la nutrition et génèrent des revenus pour de nombreuses populations rurales (Magbagbeola et al., 2010). La plante est classée parmi les cultures les plus négligées et sous-utilisées (Ipgri, 2001) et est largement remplacée par des espèces plus productives et plus rentables, comme le niébé, l'arachide et de nos jours le soja (Kouelo et al., 2012). Elle est en cours de disparition car son rendement et son aptitude au stockage sont médiocres (Borus et Bosch, 2007). Sa culture est réservée aux personnes âgées alors qu'elle nécessite une quantité de travail de sol importante (Achigan et al., 2003). En outre, aucun programme raisonné n'a été mené pour améliorer la 
productivité de la plante ni pour en étudier la variabilité génétique globale (Pasquet et al.,2015). C'est pourquoi, elle n'occupe pas une place particulière en Afrique comme d'autres cultures vivrières (arachide, maïs, niébé, riz...). Malgré l'existence des échanges entre pays voisins de l'Afrique de l'Ouest, son importance économique reste faible. Le rendement est faible et sa culture est pour la consommation locale. Le comportement vis-àvis d'un stress hydrique est inconnu. Très peu d'études relatives aux paramètres biochimiques de la lentille de terre ont été réalisées (Nguinambaye, 2010). Le choix de cette espèce pour notre étude se justifie par le fait que c'est une espèce rare qui est en voie de disparition.

Dans l'objectif de mettre en évidence d'une part, les principaux constituants biochimiques dans des différents organes de la lentille de terre; d'autre part, déterminer quelques paramètres physiologiques et agronomiques de la lentille de terre soumise à des stress hydriques variés. Pour cela, un seul essai a été installé à l'Université de Ouagadougou pour une durée de 4 mois.

\section{MATERIEL ET METHODES}

\section{Site expérimental}

L'essai a été réalisé dans la serre de l'Unité de Formation et de Recherche des Sciences de la Vie et de la Terre située dans l'enceinte de l'Université de Ouagadougou du 25 juin au 28 octobre 2009.

\section{Matériel végétal}

L'étude a porté sur une variété de la lentille de terre dont les graines collectionnées, proviennent du laboratoire d'Écophysiologie végétale.

\section{Variantes de stressage}

Les plants ont été soumis aux trois niveaux d'alimentation hydrique ci-dessous au cours de leur croissance :

$\mathrm{T}_{0}$ : témoin non stressé, arrosé chaque $48 \mathrm{~h}$ à la capacité au champ du sol ;

$\mathrm{T}_{1}$ : niveau de stress 1 , arrosage chaque 7 jours à la capacité au champ du sol ;
$\mathrm{T}_{2}$ : niveau de stress 2 , arrosage chaque 12 jours à la capacité au champ du sol.

\section{Dispositif expérimental}

L'essai a comporté 72 pots disposés en trois blocs. Chaque bloc était composé de 24 pots totalement randomisés.

\section{Semis}

Avant le semis, les graines ont été triées puis deux (2) graines ont été semées dans chaque pot à une profondeur de $3 \mathrm{~cm}$. Deux semaines après le semis, le démariage a été effectué pour laisser un plant par pot et un repiquage a été également mené pour compenser le vide observé dans certains pots.

\section{Début du traitement des stress}

Pour permettre aux plants d'acquérir une certaine capacité de résistance, les différents stress ont été appliqués 3 semaines après semis.

\section{Traitement chimique}

La terre contenue dans les pots a été traitée au préalable au furagan pour lutter contre les vers de terre et d'autres agents vecteurs des maladies. Vingt (20) jours après le semis, un traitement avec le décis a été appliqué aux plants.

\section{Méthodes d'extraction et dosage des protéines totales, des sucres totaux et des sucres réducteurs}

Les paramètres biochimiques ont été étudiés dans une première phase au niveau des feuilles, tiges et racines de la lentille de terre durant la période du traitement hydrique. Il s'agissait de déterminer la teneur en sucres totaux, en sucres réducteurs et en protéines totales. En effet, on a procédé à l'échantillonnage en un temps précis et régulier au cours de la journée entre $12 \mathrm{~h}$ et $14 \mathrm{~h}$. Plusieurs prélèvements ont été effectués pendant toute la période de stress. Les échantillons prélevés ont été pesés pour avoir le poids frais (Pf). Le poids sec (Ps) a été obtenu après le passage des échantillons à l'étuve à $80^{\circ} \mathrm{C}$ pendant $24 \mathrm{~h}$. Les différents 
résultats ont été obtenus en faisant la moyenne arithmétique des valeurs trouvées.

Pendant la récolte, les gousses ont été collectées par plante. Après séchage, ces gousses ont été décortiquées pour recueillir les graines. Les différentes variables mesurées sur les gousses et les graines ont permis d'estimer les différentes composantes du rendement et de déterminer le rendement en graines. Les valeurs moyennes ont été regroupées dans des tableaux

Toutes les analyses ont été faites avec la matière sèche (MS) obtenue à partir des différents organes de la plante.

- La détermination des protéines totales a été faite par la méthode de (Bradford, 1976). La concentration protéique a été calculée à partir de la courbe d'étalonnage établie avec la protéine standard. Les densités optiques ont été lues à $620 \mathrm{~nm}$ et $450 \mathrm{~nm}$.

- La détermination des sucres totaux a été faite par la méthode de (Fox et Robyl, 1991). La concentration en sucres a été calculée à partir d'une courbe d'étalonnage établie avec le glucose comme sucre de référence. Les densités optiques ont été lues à 492 nm.

- La détermination des sucres réducteurs a été faite selon la méthode de (Miller, 1958). La concentration en sucres réducteurs a été obtenue à partir d'une courbe d'étalonnage établie avec le glucose comme sucre standard. Les densités optiques ont été lues à $546 \mathrm{~nm}$.

\section{La détermination de la chlorophylle}

La méthode de Mackney (1941) a permis de déterminer la teneur de chlorophylle des feuilles. Il s'agit de broyer $100 \mathrm{mg}$ de matière fraîche (MF) en présence d'acétone à $80 \%$. Après filtration, la densité optique a été lue à 663 et $645 \mathrm{~nm}$. Les concentrations en chlorophylle ont été obtenues à partir des formules suivantes :

$[\mathrm{Chl} \mathrm{A}]=12(\mathrm{DO} 663)-2,67(\mathrm{DO} 645)$

$[\mathrm{Chl} \mathrm{B}]=22,5($ DO 645) - 2, 65 (DO 663)

[Chl A] : Concentration de la chlorophylle A en $\mathrm{mg} / \mathrm{MF}$
[Chl B] : Concentration de la chlorophylle B en $\mathrm{mg} / \mathrm{MF}$

\section{Analyses statistiques}

Les données ont fait l'objet d'une analyse de variance avec le logiciel XL STAT 7.5.2 version 2004. Les moyennes ont été comparées en utilisant le test de Fisher au seuil de $5 \%$.

\section{RESULTATS}

\section{Taille moyenne des plants}

Le Tableau1 présente l'effet du stress sur la taille des plants. La taille des plantes témoins est plus élevée $(6,88$ a $\pm 0,44)$ comparativement à celle des plantes stressées $\left(5,52^{\mathrm{b}} \pm 0,15\right)$. L'analyse statistique des moyennes de taille a donné des résultats dont les différences ont été très hautement significatives entre les plantes en alimentation hydrique normale et celles stressées. Par contre, d'un niveau de stress à un autre, il n'y a pas une différence significative.

\section{Poids de la biomasse sèche}

Le Tableau 2 présente l'effet dépressif du stress sur le poids de la biomasse sèche. Les plantes à régime hydrique non limitant, pour le cas des feuilles, ont produit une biomasse sèche double $(21,6 \%)$ par rapport à celles en condition de stress modéré $(11 \%)$ et 4 fois celles des plantes plus stressées $(5,3 \%)$. Ces résultats sont également semblables pour les racines et les tiges. Les plantes en condition de stress plus sévère ont un poids de biomasse plus faible pour tous les organes. D'un régime hydrique à un autre, il ya une variation significative.

\section{Début de la floraison}

Chez les plantes à régime hydrique non limité, les premiers boutons floraux ont apparu 50 jours après le semis. Les conditions de stress ont retardé de 5 jours le début de la floraison pour les plantes en condition stressante modérée et de 7 jours pour les plantes en stress sévère. 


\section{Durée de cycle}

Nos résultats ont montré que l'effet de stress n'a pas trop affecté le cycle des plants soumis aux différents traitements hydriques. Ainsi, les plantes à régime hydrique normal ont eu leurs boutons floraux 50 jours après le semis et ont connu un cycle de 112 jours. Les plantes sous stress hydrique modéré ont eu leurs boutons floraux 55 jours après semis et un cycle de 121 jours. Enfin, pour les plantes sous stress sévère, les premiers boutons floraux ont été observés 57 jours après semis et un cycle prolongé de 125 jours.

\section{Teneurs en chlorophylle des feuilles}

Les observations en serre au stade 63 jours après semis, ont révélé que les feuilles des plantes témoins sont de couleur vert-clair. Les feuilles des plantes à déficit hydrique au contraire, sont de couleur vert-foncé (celles à stress sévère sont encore plus foncées). Le Tableau 3 montre que la teneur en chlorophylle des feuilles des plantes en conditions de stress sévère est plus élevée $(16,01 \%)$ en comparaison avec celle des feuilles des plants sous stress léger $(12,43 \%)$. Par contre, les plantes témoins ont une teneur faible en chlorophylle $(9,91 \%)$.

\section{Comparaison de la taille des gousses par traitement hydrique}

Les gousses obtenues après la récolte sont de tailles très variables. Cette variation de taille s'observe aussi bien à l'intérieur d'un régime hydrique que d'un régime hydrique à un autre. La Figure 1 montre que les plantes sévèrement stressées présentent des gousses de taille 2 fois plus grosses $(3,5 \mathrm{~cm})$ que celles des plantes témoins $(1,8 \mathrm{~cm})$. La taille moyenne des gousses des plantes sous stress léger est de $2,3 \mathrm{~cm}$. Les plantes les plus stressées ont de gousses de poids moyen $0,36 \mathrm{~g}$ alors que les plantes témoins ont des gousses poids moyen de $0,101 \mathrm{~g}$. Celui des plantes moyennement stressées est de $0,210 \mathrm{~g}$.

\section{Taux de sucres totaux dans les différentes parties de la lentille de terre}

Pour les plantes témoins, le taux de sucres totaux (TST) est élevé dans la racine, la tige et la feuille par rapport au TST dans ces différentes parties des plantes stressées
(Tableau 4). Ce qui est contraire dans la graine où ce sont les plantes stressées qui ont un TST élevé.

\section{Teneurs racinaires en sucres totaux}

On remarque que quelle que soit la durée du stress hydrique, le TST diminue en fonction du régime hydrique. Ainsi, ce taux est faible $(12,93 \%)$ pour les plantes stressées et élevé $(20,54 \%)$ pour les plantes témoins. Un effet très hautement significatif des traitements hydriques sur le taux d'accumulation des sucres totaux a été décelé par l'analyse de variance tout au long du stress (Tableau 4).

\section{Teneurs en sucres totaux des tiges}

Le Tableau 4 montre un TST un peu élevé $\left(9,76^{\mathbf{d}} \pm 0,12\right)$ dans des tiges de la plante en condition d'alimentation hydrique normale comparativement aux plantes stressées $\left(7,7^{\mathrm{e}} \pm\right.$ $0,04)$. Il y a une différence significative d'effets des traitements hydriques sur le taux d'accumulation des sucres totaux des tiges des plantes témoins et des plantes stressées. Mais d'un niveau de stress à un autre, la différence n'est pas significative (Tableau 4).

\section{Teneurs foliaires des sucres totaux}

Dans les feuilles, les plantes à régime hydrique normal ont présenté un taux d'accumulation élevé de sucres totaux $\left(16,07^{\mathrm{f}}\right.$ $\pm 0,5)$ par rapport à celles stressées $\left(9,90^{\mathbf{h}} \pm\right.$ $0,35)$. L'analyse statistique révèle un effet significatif des traitements hydriques sur le taux d'accumulation de sucres totaux entre les différents traitements hydriques.

\section{Taux de sucres totaux des graines}

Dans le tableau 4, nous remarquons que les graines des plantes à régime hydrique plus stressant présentent des TST très élevés $\left(24,65^{\mathbf{j}}\right.$ $\pm 0,1$ ), pratiquement 2 fois par rapport aux graines des plantes à régime hydrique non limitant $\left(11,3^{\mathbf{i}} \pm 0,1\right)$ et moyennement stressant $\left(12,73^{\mathbf{i}} \pm 0,2\right)$.

Taux de sucres réducteurs (TSR) dans les différentes parties de la lentille de terre

Le Tableau 5 montre le taux des sucres réducteurs (TSR) dans les différents organes de la lentille de terre. Selon ce tableau, le TSR est plus élevé dans les organes des plantes 
comparativement aux plantes stressées. Cette situation est inverse dans le cas de la graine.

\section{Teneurs racinaires en sucres réducteurs}

Le taux d'accumulation des sucres réducteurs dans les racines des plantes témoins est plus élevé $\left(8,3^{\mathrm{a}} \pm 0,1\right)$ par rapport à celui des plantes stressées qui est faible $\left(6,52^{\mathbf{b}} \pm\right.$ 0,12 ) (Tableau 5).

\section{Teneurs en sucres réducteurs des tiges}

Le TSR des plantes moyennement stressées est plus élevé $\left(3,24^{\mathbf{d}} \pm 0,01\right)$ dans les tiges. Dans ce cas précis, les plantes plus stressées ont un TSR plus faible $\left(2,47^{\mathfrak{c}} \pm 0,11\right)$. La variation des TSR des tiges sous l'effet des traitements hydriques est significative (Tableau 5).

\section{Teneurs foliaires en sucres réducteurs}

Les taux d'accumulation des sucres réducteurs des feuilles des plantes témoins $\left(5,6^{\mathrm{e}} \pm 0,12\right)$ et celles moyennement stressées $\left(5,7^{\mathrm{e}} \pm 0,3\right)$ ne sont pas différents. Cette variation des TSR des feuilles n'est pas soumise à un effet très significatif des traitements hydriques appliqués (Tableau 5).

\section{Teneurs en sucres réducteurs des graines}

Au niveau des graines, les plantes en conditions stressantes, présentent des taux d'accumulation en sucres réducteurs plus élevés $\left(5,97^{\mathrm{g}} \pm 0,03\right)$ par rapport à celles soumises à un régime hydrique non limitant $\left(3,18^{\mathrm{f}} \pm 0,02\right)$. L'effet est hautement significatif sur le taux d'accumulation des sucres réducteurs des plantes témoins et des plantes stressées selon l'analyse de variance. Mais d'un niveau de stress à un autre, cet effet n'est pas significatif (Tableau 5).
Il y a une corrélation positive $(\mathrm{R}=0,72)$ entre les sucres totaux et les sucres réducteurs dans les différents organes de la lentille de terre. Ainsi, l'augmentation de la teneur en sucres réducteurs entraîne celle des sucres totaux.

Taux de protéines totales (TPT) dans les différentes parties de la lentille de terre Teneurs en protéines totales des tiges

Les teneurs en protéines totales des tiges de la lentille de terre évoluent en fonction des différents régimes hydriques. Les taux d'accumulation des protéines totales des tiges sont plus élevés $\left(4,98^{\mathrm{a}} \pm 0,21\right) \mathrm{chez}$ les plantes à régime non limitant par rapport aux plantes stressées $\left(1,68^{\mathrm{b}} \pm 0,11\right)$. L'analyse statistique révèle que l'effet des traitements hydriques appliqués est hautement significatif par rapport au taux d'accumulation de protéines totales des tiges (Tableau 6).

\section{Teneurs foliaires en protéines totales}

Les teneurs en protéines totales des feuilles des plantes témoins sont élevées $\left(12^{\circ}\right.$ $\pm 0,32)$ par rapport à celles des plantes stressées $\left(8,79^{\mathrm{e}} \pm 0,1\right)$. Il existe un effet très significatif des taux d'accumulation des protéines totales des feuilles (Tableau 6).

\section{Teneurs en protéines totales des graines}

Les teneurs en protéines totales des graines évoluent en fonction du régime hydrique. Le taux de protéines plus faible $\left(9,7^{\mathrm{f}}\right.$ $\pm 0,1)$ chez les plantes témoins devient plus élevé chez les plantes stressées $\left(13,43^{\mathrm{h}} \pm 0,2\right)$. Cette évolution des taux de protéines totales dans les graines sous différents stress a un effet très significatif (Tableau 6).

Tableau 1 : Taille moyenne des plants en fonction des traitements.

\begin{tabular}{|c|c|c|c|c|}
\hline Traitement & & & $\mathrm{cm}$ & \\
\hline Bloc & $\mathbf{B}_{1}$ & $\mathbf{B}_{2}$ & $\mathbf{B}_{3}$ & Moyenne \\
\hline $\mathrm{T}_{0}$ & $6,5 \pm 0,9$ & $6,6 \pm 1,1$ & $7,5 \pm 1,1$ & $6,88^{a} \pm 0,44$ \\
\hline $\mathrm{T}_{1}$ & $6,05 \pm 0,58$ & $6,38 \pm 0,78$ & $6,69 \pm 1,09$ & $6,02^{a} \pm 0,31$ \\
\hline $\mathrm{T}_{2}$ & $5,31 \pm 0,93$ & $5,63 \pm 1,1$ & $5,63 \pm 1,0$ & $5,52^{b} \pm 0,15$ \\
\hline
\end{tabular}

La taille moyenne des plants est exprimée sous la forme d'une moyenne \pm écart-type. Les moyennes dans la dernière colonne suivies des lettres différentes ( $\mathrm{a}$ et $\mathrm{b})$ sont significativement différentes $(\mathrm{P}<0,005)$. 
Tableau 2 : Pourcentage du poids de la biomasse sèche par régime hydrique après deux cycles de stress.

\begin{tabular}{ccccccccc}
\hline & Racine (\%) & \multicolumn{3}{c}{ Tige (\%) } & \multicolumn{3}{c}{ Feuille (\%) } \\
\hline $\mathrm{T}_{0}$ & $\mathrm{~T}_{1}$ & $\mathrm{~T}_{2}$ & $\mathrm{~T}_{0}$ & $\mathrm{~T}_{1}$ & $\mathrm{~T}_{2}$ & $\mathrm{~T}_{0}$ & $\mathrm{~T}_{1}$ & $\mathrm{~T}_{2}$ \\
15 & 7,5 & 3,8 & 17,7 & 9 & 4,4 & 21,6 & 11 & 5,3 \\
\hline
\end{tabular}

Tableau 3 : Teneur en chlorophylle pour $100 \mathrm{mg}$ de feuilles fraîches au stade 63 jours après semis.

\begin{tabular}{lccc}
\hline Traitement & $\mathbf{T}_{\mathbf{0}}$ & $\mathbf{T}_{\mathbf{1}}$ & $\mathbf{T}_{\mathbf{2}}$ \\
\hline Teneur en chlorophylle A pour 100 mg/MF & 9,91 & 12,43 & 16,01 \\
Teneur en chlorophylle B pour 100 mg/MF & 7,85 & 1,44 & 12,99 \\
\hline
\end{tabular}

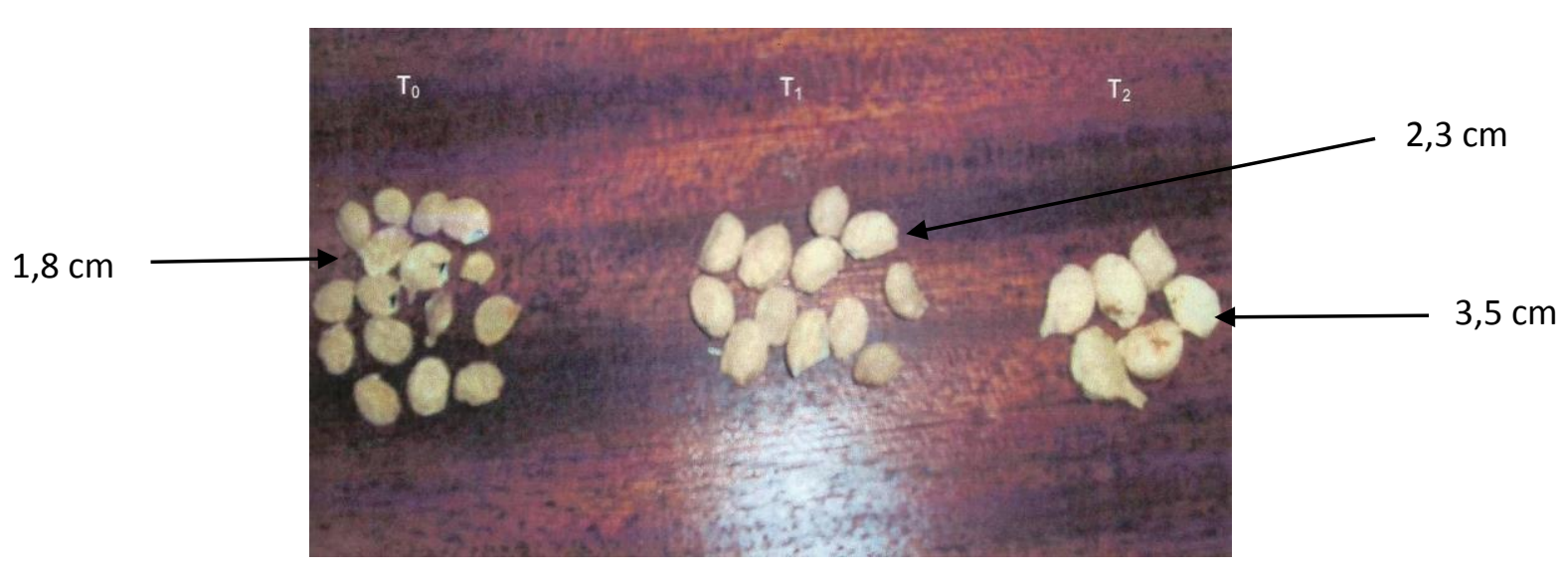

Figure 1 : Taille des gousses en fonction des traitements hydriques.

Tableau 4 : Taux moyens de sucres totaux dans les différentes parties de la lentille de terre.

\begin{tabular}{cccc}
\hline Traitement TST $(\%)$ des organes & $\mathbf{T}_{\mathbf{0}}$ & $\mathbf{T}_{\mathbf{1}}$ & $\mathbf{T}_{\mathbf{2}}$ \\
Racine & $20,54^{\mathbf{a}} \pm 0,1$ & $15,53^{\mathbf{b}} \pm 0,2$ & $12,93^{\mathbf{c}} \pm 0,05$ \\
Tige & $9,76^{\mathbf{d}} \pm 0,12$ & $8,37^{\mathbf{d}} \pm 0,3$ & $7,7^{\mathbf{e}} \pm 0,04$ \\
Feuille & $16,07^{\mathbf{f}} \pm 0,5$ & $12,73^{\mathbf{g}} \pm 0,04$ & $9,90^{\mathbf{h}} \pm 0,35$ \\
Graine & $11,3^{\mathbf{i}} \pm 0,1$ & $12,73^{\mathbf{i}} \pm 0,2$ & $24,65^{\mathbf{j}} \pm 0,1$ \\
\hline
\end{tabular}

Les taux moyens des sucres totaux dans les différentes parties de la lentille de terre sont exprimés sous la forme d'une moyenne \pm écart-type. Les moyennes dans les colonnes suivies des lettres différentes sont significativement différentes $(\mathrm{P}<0,005)$. 
Tableau 5 : Taux moyens de sucres réducteurs dans les différentes parties de la lentille de terre.

\begin{tabular}{lccc}
\hline Traitement TSR(\%) des organes & & & \\
& $\mathbf{T}_{\mathbf{0}}$ & $\mathbf{T}_{\mathbf{1}}$ & $\mathbf{T}_{\mathbf{2}}$ \\
\hline Racine & $8,3^{\mathrm{a}} \pm 0,1$ & $7^{\mathrm{a}} \pm 0,15$ & $6,52^{\mathrm{b}} \pm 0,12$ \\
Tige & $2,78^{\mathrm{c}} \pm 0,2$ & $3,24^{\mathrm{d}} \pm 0,01$ & $2,47^{\mathbf{c}} \pm 0,11$ \\
Feuille & $5,6^{\mathrm{e}} \pm 0,12$ & $5,7^{\mathrm{e}} \pm 0,3$ & $4,94^{\mathrm{e}} \pm 0,1$ \\
Graine & $3,18^{\mathrm{f}} \pm 0,02$ & $5,7^{\mathrm{g}} \pm 0,15$ & $5,97^{\mathrm{g}} \pm 0,03$ \\
\hline
\end{tabular}

Dans une même ligne, les chiffres suivis d'une même lettre sont statistiquement identiques au seuil de 5\% (test de Fisher). Moyenne \pm écart-type.

Tableau 6 : Taux moyens de protéines totales dans les différentes parties de la lentille de terre.

\begin{tabular}{lccc}
\hline Traitement TPT $(\%)$ des organes & $\mathbf{T}_{\mathbf{0}}$ & $\mathbf{T}_{\mathbf{1}}$ & $\mathbf{T}_{\mathbf{2}}$ \\
\hline Tige & $4,98^{\mathrm{a}} \pm 0,21$ & $2,73^{\mathrm{b}} \pm 0,26$ & $1,68^{\mathrm{b}} \pm 0,11$ \\
Feuille & $12^{\mathrm{c}} \pm 0,32$ & $10,30^{\mathrm{d}} \pm 0,3$ & $8,79^{\mathrm{e}} \pm 0,1$ \\
Graine & $9,7^{\mathrm{f}} \pm 0,1$ & $11,4^{\mathrm{g}} \pm 0,12$ & $13,43^{\mathrm{h}} \pm 0,2$ \\
\hline
\end{tabular}

Taux moyens de protéines totales dans les différentes parties de la lentille de terre sont exprimés sous la forme d'une moyenne \pm écart-type. Les moyennes dans les colonnes suivies des lettres différentes sont significativement différentes $(\mathrm{P}<0,005)$.

\section{DISCUSSION}

Les plantes à régimes hydriques stressants sont de taille réduite par rapport à celle des plantes témoins puisque le stress hydrique est un facteur de réduction de la tige des plantes. En effet, le stress hydrique par réduction de la surface foliaire est à l'origine de la réduction de la capacité photosynthétique des plantes. Les pertes d'eau par transpiration, aussi minime soient-elles, peuvent provoquer la turgescence des cellules des différents organes. Ainsi, les plantes stressées ne sont pas bien développées. Elles sont moins évoluées et la croissance de la partie aérienne est réduite. Le nombre des tiges par défaut, l'élongation des tiges, le nombre des entre-nœuds et des ramifications sont tous réduits par le stress hydrique. En effet, la croissance est le premier indicateur de l'état nutritionnel des plantes. C'est un indicateur de la disponibilité des éléments nutritifs et leur utilisation par la plante. Une déficience quelconque en éléments nutritifs peut diminuer le développement de la plante (Bado, 2002).

Les plantes à régimes hydriques stressants ont des organes produisant une biomasse sèche plus faible que ceux des plantes témoins. Cela s'explique par le fait que l'intensité du stress hydrique a exercé des effets dépressifs par exemple une perte de biomasse. Adjal (2006) corrobore en prouvant que les plantes stressées produisent moins de biomasse sèche en comparaison avec celles soumises à un régime hydrique non limitant. Dans le même ordre, Diallo (2003), travaillant sur le riz, a trouvé que les plantes stressées ont un poids deux fois plus faible que celui des plantes témoins. En ce qui concerne les graines, nos travaux ont montré des résultats contraires. Les graines des plantes en condition de régime hydrique non limitant produisent une biomasse plus faible comparativement à celles en condition de stress. Ceci pourrait s'expliquer par le fait que les graines sont des organes de réserve donc à leur niveau il y a une forte accumulation de composés glucidiques et protéiques. La perte de la biomasse sèche sous l'effet dépressif du stress hydrique dans ce cas a été moindre.

Le stress hydrique appliqué lors de l'essai, a contribué aux troubles physiologiques, à savoir la teneur en chlorophylle des feuilles, le décalage du début de floraison et les cycles des plantes. Ainsi, la coloration vert-foncé des feuilles stressées pourrait s'expliquer par le fait qu'il y a une 
forte concentration de la chlorophylle dans ces feuilles due au déficit hydrique. Notons que la chlorophylle est une molécule grâce à la quelle, la plante est capable de réaliser la photosynthèse c'est-à-dire la fabrication des glucides à partir des molécules minérales sous l'action de la lumière visible. Alors, la forte concentration de la chlorophylle dans les feuilles des plantes à régime hydrique stressé pourrait être à l'origine d'une importante photosynthèse. Ce qui conduirait à une forte accumulation glucidique dans les graines. Les plantes témoins par contre, bien arrosées subissent un phénomène de dilution. Dans ce cas, il ya une bonne circulation d'eau et ainsi, la chlorophylle ne se concentre pas au niveau des feuilles. C'est pourquoi, elles ont une coloration vert-clair (Nguinambaye, 2010). Bougdad (2015) a montré que l'augmentation de la teneur en chlorophylle notée chez les populations de Janet et Chott quand elle est moins arrosée, serait probablement la conséquence de la réduction de la taille des cellules foliaires sous l'effet du stress hydrique qui engendre une plus grande concentration. Cette hypothèse est renforcée par la réduction de teneur relative en eau des feuilles et de la surface foliaire chez ces populations.

Le début de la floraison des plantes a varié en fonction des traitements. Ainsi, les premiers boutons sont apparus 50 jours après semis chez les plantes à régime hydrique non limitant. Ces résultats sont contraires à ceux de Tiene (2015) qui ont montré que c'est au $60^{\text {ème }}$ jours après semis (JAS) que l'on voit apparaître les premiers boutons floraux à l'aisselle de certaines feuilles de la lentille de terre. Cette différence peut s'expliquer dans les conditions de culture et surtout la quantité des rayonnements lumineux reçue par nos plantes. Les conditions de stress ont retardé le début de la floraison pour les plantes en condition de stress modérée de 5 jours et celles sévères de 7 jours. Ce qui nous amène à conclure que le stress a provoqué le décalage de floraison entre les différents traitements mais ces différences ne sont pas significatives.

Le cycle cultural des plantes témoins est de 112 jours, celui des plantes à stress hydrique léger est de 121 jours puis sévère 125 jours. Ces cycles coïncident avec le cycle normal de la lentille de terre, sans stress hydrique, qui est montré par les travaux antérieurs. En effet, Brink et Belay (2006) ont montré que les gousses de la lentille de terre atteignent leurs maturités entre 40-60 jours après l'ouverture des fleurs. La durée du cycle cultural est de 90-180 jours. Par conséquent, le degré de stress choisi n'est pas suffisant pour influencer le début de floraison des plantes stressées. Il en est de même pour le cycle cultural. Les différences des durées des cycles ne sont pas significatives. Le stress hydrique appliqué n'a pas un degré suffisant pour affecter les cycles des plantes stressées.

La grosseur des gousses obtenues varie en fonction des différents traitements hydriques appliqués. Les gousses des plantes plus stressées sont de plus en plus grosses par rapport à celles des plantes témoins à cause de l'accumulation dans les graines des composés glucidiques et protéiques. Face à ce stress hydrique, les plantes stressées ont tendance à réagir par l'augmentation de quantités de sucres et de protéines totales de leurs cellules. C'est ainsi que Libbey (2003) affirme que la plupart des plantes augmentent, sous l'effet de la sécheresse, leur concentration en solutés (ions inorganiques, acides aminés sucres solubles, acides organiques, azote soluble). Comme conséquence de cette concentration de solutés dans les graines, les plantes stressées produisent des gousses de grande taille, matures, bien remplies, de bonne qualité dont les graines ont un poids élevé.

Les différents résultats biochimiques ont montré que les taux des hydrates de carbone et des protéines sont élevés dans les racines et les feuilles alors que ceux-ci ont baissé dans les tiges. Ceci trouverait son explication dans le rôle de mobilisation que jouent les racines. En effet, les racines sont des organes mobilisateurs d'éléments nécessaires à la nutrition des plantes. Par ailleurs, les feuilles sont des organes de synthèse d'éléments organiques. Pour cette raison, Zufferey en 2012, les appelle organes sources. Les tiges quant à elles, sont des organes de transit par lesquels les éléments mobilisés au niveau des 
racines circulent pour atteindre l'organe de synthèse.

La réduction des taux des composés glucidiques et protéiques chez les plantes en conditions de stress par rapport aux plantes témoins pourrait s'expliquer par la perte de la biomasse. Cette biomasse est aussi constituée des substances organiques. Par conséquent, sa perte entraine aussi une diminution du taux des composés organiques. Par ailleurs, cette diminution de la biomasse serait due à la baisse du métabolisme de la plante. En effet, les plantes plus stressées ont un nombre réduit de feuilles donc en conséquence, elles produisent une faible quantité de biomasse à cause de leur photosynthèse qui est réduite. Dans les graines au contraire, les résultats ont montré une hausse significative des teneurs des composés glucidiques et protéiques comparativement aux plantes témoins. Ceci trouverait son explication dans le fait que les graines sont des organes de réserve (Libbey, 2003). L'accumulation des substrats carbonés et protéiques dans les graines lors des stress montre que la plante accorde une priorité aux graines lors du déficit hydrique. Selon Badiel (2013), la valeur énergétique de la graine de lentille de terre est estimée à $354,06 \mathrm{Kcal}$ pour $100 \mathrm{~g}$ du poids sec. Tout est fait comme si la plante voudrait préserver les organes vitaux pour sauvegarder l'espèce.

Malgré les stress appliqués, les plantes en serre se sont adaptées et ont évolué pour atteindre la maturité et produire les gousses. La variation des taux d'hydrates de carbone d'un organe à l'autre serait à l'origine de la résistance de ces plantes face aux troubles physiologiques causés par le déficit hydrique. De ce fait, les mécanismes de variation des taux des glucides dans les différents organes pourraient être une forme d'adaptation de la plante face aux difficultés telles que la sècheresse. Ces résultats sont conformes à ceux de Bazot (2005). Ainsi, la variation des taux des glucides offre un moyen à la plante d'ajuster sa croissance par la fourniture du carbone en situation de contraintes ou des changements environnementaux. Cette idée est partagée aussi par Faouzi (2007) qui affirme que les produits carbonés peuvent alors être utilisés, autant pour l'ajustement osmotique que pour la croissance racinaire. En effet, de nombreux auteurs s'accordent à dire que les glucides fournissent du carbone à la plante face à des contraintes et des changements environnementaux (Farrar et Jones, 2000).

La restitution des hydrates de carbone des racines, des tiges et des feuilles aux graines souterraines n'a pas été totale tant chez les plantes stressées que celles témoins (Nguinambaye, 2010). En présence de la lumière solaire, les plantes utilisent l'eau et le dioxyde de carbone pour créer les hydrates de carbone. La plante utilise les hydrates de carbone comme source d'énergie pour sa croissance. Quand la croissance de la plante ralentit et qu'elle produit plus d'hydrates de carbone que pour ses besoins de croissance, l'excédent est mis en réserve (Farrar et Jones, 2000). Ces réserves sont importantes pour assurer la survie de la plante. La restitution des hydrates carbonés aux graines ne peut être totale puis que ces organes (feuilles, tiges, racines) ont besoin de survivre. Il leur faut ces hydrates qui constituent une source d'énergie. Si la restitution avait été totale, les cellules de ces organes connaitraient un déséquilibre, ce qui compromettrait le fonctionnement de la plante (Nguinambaye, 2010). Nos résultats ont montré que les composés protéiques sont plus restitués aux graines que les substrats carbonés. C'est pour cela que les graines de la lentille de terre sont très riches en protéines totales (Nguinambaye, 2010). Les résultats antérieurs ont montré que dans ses aires de culture, les consommateurs préfèrent les graines de Macrotyloma geocarpum à celles de Vigna unguiculata et de Vigna subterranea en raison de leur appétence et présumée haute teneur en protéines (Baudoin et Mergeai, 2001).

\section{Conclusion}

Cette étude a pour but de mettre en évidence quelques paramètres physiologiques et quelques constituants biochimiques dans les différents organes de la lentille de terre soumise à des stress hydriques. Au terme de cette étude, il ressort des résultats que le stress hydrique varié appliqué, a affecté les plantes sur tous les aspects. Ainsi, des troubles 
physiologiques importants ont été constatés. Les feuilles plus stressées ont accumulé une forte concentration de chlorophylle. Les éléments biochimiques sont abondants dans les racines, les feuilles et les graines mais leur taux est faible dans les tiges. Le taux des constituants biochimiques se distingue aussi en fonction du régime hydrique. Le taux est élevé chez les plantes en comparaison avec celui des plantes stressées. La lentille de terre est une plante bien adaptée au climat tropical. En perspective, nous envisageons soumettre des lentilles de terre en conditions de stress variés pour mesurer le degré de tolérance à la sécheresse et aussi étudier la conductance stomatique.

\section{CONFLIT D'INTERETS}

Les auteurs de cet article trouvent qu'il n'y a aucun conflit d'intérêts.

\section{CONTRIBUTIONS DES AUTEURS}

Les collègues, co-auteurs ont pris part activement à la réalisation de cette étude. Certains ont contribué à la mise en place de l'essai et ont suivi l'évolution des plantes soumises aux stress ; d'autres ont participé aux corrections du manuscrit.

\section{REMERCIEMENTS}

Nous tenons à remercier l'Université de Ndjamena et l'Université de Ouagadougou qui ont soutenu matériellement et moralement ce travail. Nous remercions aussi les collègues qui ont pris part activement à la réalisation de cet article.

\section{REFFERENCES}

Achigan Dako GE, Vodouhè SR, Koukè A. 2003. Collecte des ressources génétiques $\mathrm{du}$ voandzou (Vigna subterranea (L.) Verde.) et $\mathrm{du}$ dohi (Macrotyloma geocarpum (Harms) Maréchal et Baudet) au Centre Bénin. In: Actes de l'atelier scientifique du Centre des Recherches Agricoles du Centre Savè, Agossou V, Amandji F, Agbo B, Tandjiékpon A (Eds). 18-19 décembre 2002, Dassa, Bénin. Institut National des Recherches
Agricoles du Bénin, Cotonou, Bénin ; 93107.

Achigan Dako GE, Vodouhè SR. 2006. Macrotyloma geocarpum (Harms) Maréchal et Baudet. In Plant Resources of Tropical Africa 1. Cereals and Pulses, Brink M, Belay G (Eds). PROTA Foundation, Backhuys PubLishers: Wageningen, Netherlands, Leiden, Netherlands/CT A, Wageningen, Netherlands; 111-114.

Adéranti FA. 2001. Morphological characterization of Kersting ground bean (Macrotyloma geocarpum (Harms) Marechal and Baudet) B. Agric. Thesis, Department of Plant Science, Obafemi Awolowo University, Ile-Ife, Nigeria, $67 \mathrm{p}$.

Adjal Hieche Y. 2006. Réponse de la luzerne (Medicago sativa L.) au stress hydrique et à la profondeur de semis. Mémoire de magistère en sciences agronomiques. Université de AL HADJLAKHDARBATNA (Algérie) $26 \mathrm{P}$.

Adu-Gyamfi R, Fearon J, Bayorbor TB, Dzomeku IK, Avornyo VK. 2011. The Status of Kersting's Groundnut (Macrotyloma geocarpum (Harms) Maréchal and Baudet) An Underexploited Legume in Northern Ghana. Outlook on Agriculture, 40(3): 259-262. DOI: https://doi.org/10.1787/19991142.

Amujoyegbe BJ, Obisesan IO, AjayiA O, AdérantiF A. 2012. Disappearance of Kersting's groundnut (Macrotyloma geocarpum (Harms) Marechal and Baudet) in south-western Nigeria: an indicator of genetic erosion. Published in Issue No.152, page 45 to 50. Publishing, Paris.

Aremu MO, Olaofe O, Akintayo ET. 2006. A comparative study on the chemical and amino acid composition of some Nigerian underutilized legume flours'. Pakistan Journal of Nutrition, 5 (1) : 34-38. DOI : https://doi.org/10.9755/ejfa.v25i7.12045

Badiel B. 2013. Constituants biochimiques et mise en évidence des caractéristiques nutritionnelles de la graine de la lentille de terre (Macrotyloma geocarpum) 
Harms. Mémoire DEA, Université de Ouagadougou $52 \mathrm{p}$.

Bado BV, Sedogo PM, Hien V, Lompo F. 2002. Utilisation efficace des engrais azotés pour une augmentation de la production vivrière: 'expérience du Burkina. In Alleviating Soil Fertility Constraints to Increased Crop Production in West Africa, Mokuwnye AU (Ed.). Kluwer Academie Publishers: Netherlands; 115-123.

Bampuori A H. 2007. Effect of Traditional Farming Practices on the Yield of Indigenous Kersting's Groundnut (Macrotyloma geocarpum Harms) Crop in the Upper West Region of Ghana. Journal of Developments in Sustainable Agriculture, 2(2): 128-144. DOI: https://doi.org/10.11178/jdsa.3.1.

Baudoin JP, Mergeai G. 2001. Kersting's groundnut. In Crop Production in Tropical Africa, Roman RH (Ed.). DGTC (Directorate General for International Cooperation), Ministry of Foreign Affairs, External Trade and International Cooperation: Brussels, Belgium; 313-317.

Bayorbor TB, Dzomeku IK, Avornyo VK, Opoku-Agyeman MO. 2010. Morphological variation in Kersting's groundnut (Kerstigiella geocarpa Harms) landraces from northem Ghana. Agriculture and Biology Journal of North America, 1(3): 290-295. DOI: 10.5251/abjna.2011.2.5.780.784.

Bazot S. 2005. Contribution à l'étude de l'allocation des photoassimilats récents dans la plante et la rhizosphère chez une graminée pérenne (Lolium perenne L.). Thèse de l'Université Paris-Sud 11, 158 p.

Bougdad KF. 2015. Effet de stress hydrique sur quelques paramètres biochimiques de la luzerne (Medicago sativa L). Mémoire Master Académique/Professionnel. UNIVERSITE KASDI MERBAH OUARGLA (Algérie), $61 \mathrm{P}$.

Borus DJ, Bosch CH. 2007. Cereals and pulses of Tropical Africa, Conclusions and recommandations based on PROTA 1: cereals and pulses. (Editeur) Wageningen: PROTA Foundation. $96 \mathrm{p}$.

Bradford MM. 1976. A rapid and sensibilive method for the quantitation of microgram quantities protein utilizing the principle of protein-dye binding. Ann. Food Journal, 6(4): 123-128. DOI: https://doi.org/10.3390/foods9050663

Brink M, Belay G. 2006. Ressources Végétales de l'Afrique Tropicale. Céréales et Légumes Secs. Fondation PROTA, Backhuys Publishers : Wageningen, Pays Bas ; $328 \mathrm{p}$.

Diallo D. 2003. Résistance à la sécheresse du riz. Criblage variétal en phase végétative. Mémoire DEA. Université de Ouagadougou $58 \mathrm{p}$.

Falalou H. 2000. Réponse physiologique du niébé (Vigna unguiculata L.W.) au déficit hydrique s'exerçant au cours de deux stades de développement, début floraison et début formation des gousses. Mémoire de DEA. Université de Ouagadougou, 62P.

Faouzi Attia 2007. Effet du stress hydrique sur le comportement écophysiologique et la maturité phrénologique de la vigne Vitis vinifera L.: Etude de cinq cépages autochtones de MIDI-PYRENEES. Thèse, Institut National Polytechnique de Toulouse, $194 \mathrm{P}$.

Farrar JF, Jones DL. 2000. The control of carbon acquisition by roots. New Phytologist, 147: 43-53. DOI: https://doi.org/10.1111/nph.16482.

Fox JD, Robyl JF. 1991. Miniaturization of three carbohydrate analyses using a micro sample plate reader. Analytical Biochemistry, 195: 93-96.

Ipgri 2001. Design and Analysis of Evaluation Trials of Genetic Resources Collections: a guide for genebank: managers, IPGRI, Rome, ltaly. 53 p.

Kouelo AF, Dadou A, Houngnandan P, Francisco MMF, Gnimassoun CJB, Sochime DJ. 2012. Impact du travail du sol et de la fertilisation minérale sur la productivité de Macrotyloma geocarpum (Harms) Maréchal et Baudet au centre du Bénin. Journal of Applied Biosciences, 
51:

3625-3632.

DOI:

10.4314/jab.v122i1.1

Kouelo AF, Azontonde A, Houngnandan P, Gangnon SAO. 2015. Effet de la fertilisation organique et minérale sur la productivité de la Lentille de Terre [Macrotyloma geocarpum (harms)] au centre du Benin. Cahiers du CBRST, Cotonou (Bénin), 1(7): Sciences de la vie. Libbey J. 2003. Le dépérissement des forêts du Maroc: analyses des causes et stratégie de lutte. Science et Changements Planétaires/Sécheresse, 14 (4): 209-18.

Mackney. 1941. Method for determination of sugars and related substances. Analytical Chemistry, 28: 350-35. DOI: https://doi.org/10.1021/ac200974w.

Magbagbeola JAO, Adetoso JA, Magbagbeola EO. 2010. Application of operations research tools to aid performance growtb of small scale industry in national development in sub-saharan Africa: case study of Nigeria. Continental Journal Social Sciences, 3: 83 - 91. DOI: http://www.wiloludjournal.com/ojs/inde x.php/cjapsci

Miller GL. 1958. Use of dinitrosalicyclic acid reagent for determination of reducing sugar. Analytical Chemistry, 31: 426-428. DOI: https://doi.org/10.1021/ac200974w Nguinambaye MM. 2010. Etude de quelques paramètres physiologiques chez la
Lentille de terre (Macrotyloma geocarpum $\mathrm{H}$.) en conditions de stress hydriques. Mémoire de DEA Université de Ouagadougou 55P.

Pasquet RS, Mergeai G, Baudoin JP. 2002. Genetic diversity of the African geocarpic legume Kersting's groundnut, Macrotyloma geocarpum (tribe Phaseoleae: Fabaceae). Biochemical Systematics and Ecology, 30: 943-952. DOI: https://doi.org/10.1016/j.bse.2020.10404 5.

Tiene K. 2015. Etude comparative des performances agronomiques de trois espèces de légumineuses a graines (Vigna unguiculata (L.) Walpers., Vigna subterranea (L.) Verdcourt., Macrotyloma geocarpum (Harms) Maréchal et Baudet) cultivées en Côte d'Ivoire. Mémoire de Master de biologie et protection des végétaux, Côte d'Ivoire, 60p.

Zufferey V, Murisier F, Vivin P, Belcher S, Lorenzini F, Spring J-L, Viret O. 2012. Reserve en glucides de la vigne (CV. Chasselas) : influence du rapport feuillefruit. Revue suisse Viticulture, Arboriculture, Horticulture, 44 (4) : 216224. 